\title{
UNIFORMLY WEAK DIFFERENTIABILITY OF THE NORM AND A CONDITION OF VLASOV
}

\author{
Dedicated to George Szekeres on his 65th birthday
}

J. R. GILES

(Received 21 October 1974)

Communicated by Jennifer Seberry Wallis

\begin{abstract}
In determining geometrical conditions on a Banach space under which a Chebychev set is convex, Vlasov (1967) introduced a smoothness condition of some interest in itself. Equivalent forms of this condition are derived and it is related to uniformly weak differentiability of the norm and rotundity of the dual norm.
\end{abstract}

In a normed linear space $X$ a set $M$ is said to be a Chebychev set if for each $x \in X$ there exists a unique nearest point $p(x) \in M$; that is,

$$
\|x-y\|>\|x-p(x)\| \text { for all } y \in M \backslash\{p(x)\} .
$$

We call the mapping $x \mapsto p(x)$ the metric projection of $X$ onto $M$.

In a rotund reflexive Banach space every closed convex set is Chebychev. But there has been considerable interest in determining conditions under which a Chebychev set is convex. Klee (1961; page 300) used uniformly weak differentiability of the norm in every direction in formulating the first characterisation of closed convex sets in terms of the Chebychev property. Vlasov (1967; page 403) showed that in a Banach space with norm uniformly weakly differentiable in every direction, and Vlasov (1970; page 778) showed that in a Banach space with rotund dual, a Chebychev set with continuous metric projection is convex.

In his earlier paper, Vlasov actually showed that a normed linear space where the norm is uniformly weakly differentiable in every direction satisfies a property which we will refer to as the Vlasov smoothness condition, (Vlasov (1967; page 402)), and it is this property which he used directly to achieve his result; we are interested in examinig the relation between uniformly weak 
differentiability of the norm in every direction, and the Vlasov smoothness condition. In his later paper Vlasov used quite different techniques; we are also interested in examining the relation between the Vlasov smoothness condition and rotundity of the dual.

In $\$ 1$ we examine uniformly weak differentiability of the norm and derive a characterisation for it in terms of the continuity of a support mapping; we use this characterisation to establish duality relations for uniformly weak differntiability of the norm. In $\$ 2$ we examine the Vlasov smoothness condition and derive equivalent reformulations for it; we use these to establish relations between smoothness, the Vlasov smoothness condition, uniformly weak differentiability of the norm, and rotundity of the dual.

I would like to thank Dr. A. L. Brown for drawing my attention to these differentiability conditions and for the correspondence we have had on the subject. I would like to thank my student Christine Paine for her critical review of the work as it has been produced.

\section{Uniformly weak differentiability of the norm}

Throughout this paper will confine our attention to real normed linear spaces. For a normed linear space $X$ we denote by $S(X)$ the unit sphere $\{x \in X:\|x\|=1\}$. We say that the norm of $X$ is

(i) weakly differentiable at $x \in S(X)$ in the direction $y \in S(X)$ if for real $\lambda$,

$$
\lim _{\lambda \rightarrow 0} \frac{\|x+\lambda y\|-\|x\|}{\lambda} \text { exists, }
$$

(ii) uniformly weakly differentiable in the direction $y \in S(X)$ if convergence to the limit in (i) is uniform for all $x \in S(X)$.

We denote the dual space by $X^{*}$, and for each $x \in S(X)$ we denote by $D(x)$ the set $\left\{f \in S\left(X^{*}\right): f(x)=1\right\}$. We denote by $D(X)$ the set $\cup\{D(x): x \in S(X)\}$. A mapping $x \rightarrow f_{x}$ of $X$ into $X^{*}$ is called a support mapping if for each $x \in S(X)$ and real $\lambda \geqq 0, f_{x} \in D(x)$ and $f_{\lambda x}=\lambda f_{x}$.

Weak differentiability of the norm can be characterised by a continuity property of the support mapping, (Giles (1971; page 107)), and uniformly weak differentiability of the norm can be similarly characterised. Cudia (1964; page 302) and Zizler (1968; page 423) have given such a characterisation of uniformly weak differentiability of the norm but ours is a local characterisation and is proved by a simpler argument following the pattern of Giles (1971; page 107).

THEOREM 1. The norm of $X$ is uniformly weakly differentiable in the direction $y \in S(X)$ if and only if there exists a support mapping $x \mapsto f_{x}$ such that the real mapping $x \mapsto f_{x}(y)$ is uniformly continuous on $S(X)$. 
Proof. Given any support mapping $x \mid \rightarrow f_{x}$ on $X$, for $x, y \in S(X)$ and real $\lambda>0$,

$$
\frac{f_{x+\lambda y}(y)}{\|x+\lambda y\|} \geqq \frac{\|x+\lambda y\|-\|x\|}{\lambda} \geqq f_{x}(y), \quad(\text { Giles (1971; page 108)). }
$$

For $\lambda<0$ these inequalities are reversed.

It is clear from these inequalities that the continuity property of the mapping implies the uniformly weak differentiability of the norm in the direction $y$.

Conversely, if the norm of $X$ is uniformly weakly differentiable in the direction $y \in S(X)$, then given $\varepsilon>0$ there exists a $\delta(\varepsilon, y)>0$ such that for every $x \in S(X)$ and every $f_{x} \in D(x)$,

$$
\left|\frac{\|x+\lambda y\|-\|x\|}{\lambda}-f_{x}(y)\right|<\varepsilon \quad \text { when } \quad \frac{1}{2} \delta<|\lambda|<\delta .
$$

Then

$$
\begin{aligned}
&\left|\left(f_{x}-f_{z}\right)(y)\right| \leqq f_{x}(y)-\frac{\|x+\lambda y\|-1}{\lambda} \mid \\
&+\left|\frac{\|x+\lambda y\|-1}{\lambda}-\frac{\|z+\lambda y\|-1}{\lambda}\right|+\left|f_{z}(y)-\frac{\|z+\lambda y\|-1}{\lambda}\right|<4 \varepsilon \\
& \text { when }\|x-z\|<\varepsilon \delta .
\end{aligned}
$$

We say that

(i) a normed linear space $X$ is weakly uniformly rotund in the direction $g \in S\left(X^{*}\right)$ if given $\varepsilon>0$ there exists a $\delta(\varepsilon, g)>0$ such that, for $x, y \in S(X)$

$$
|g(x-y)|<\varepsilon \quad \text { when }\|x+y\|>2-\delta
$$

(ii) a dual space $X^{*}$ is weak ${ }^{*}$ uniformly rotund in the direction $y \in S(X)$ if given $\varepsilon>0$ there exists a $\delta(\varepsilon, y)>0$ such that, for $f, g \in S\left(X^{*}\right)$

$$
|(f-g)(y)|<\varepsilon \text { when }\|f+g\|>2-g .
$$

For a Banach space $X$, Šmulian (1940; page 645) established duality relations between uniformly weak differentiability of the norm in $X$ and weak* uniform rotundity of $X^{*}$, and also between uniformly weak differentiability of the norm in $X^{*}$ and weakly uniform rotundity of $X$. We establish local duality relations using the characterisation of uniformly weak differentiability of the norm given in Theorem 1; our argument is an adaptation of Giles (1971; page 111). Cudia (1964; page 295) has also given a proof of these local duality relations but our argument using Theorem 1 is again much simpler.

THeOREм 2. (i) The norm of $X$ is uniformly weakly differentiable in the direction $y \in S(X)$ if and only if $X^{*}$ is weak ${ }^{*}$ uniformly rotund in the direction $y$; 
(ii) for a Banach space $X$, the norm of $X^{*}$ is uniformly weakly differentiable in the direction $g \in S\left(X^{*}\right)$ if and only if $X$ is weakly uniformly rotund in the direction $\mathrm{g}$.

Proof. (i) Given any support mapping $x \mapsto f_{x}$ on $X$ the following primitive parallelogram law holds,

$$
2\left(\|x\|^{2}+\|z\|^{2}\right) \leqq\|x+z\|\left\|f_{x}+f_{z}\right\|+\|x-z\|\left\|f_{x}-f_{z}\right\|
$$

for all $x, z \in X$.

When $X^{*}$ is weak ${ }^{*}$ uniformly rotund in the direction $y \in S(X)$ then, given $\varepsilon>0$ there exists a $\delta(\varepsilon, y)>0$ such that, for $f_{x}, f_{z} \in S\left(X^{*}\right)$

$$
\left|\left(f_{x}-f_{z}\right)(y)\right|<\varepsilon \text { when }\left\|f_{x}+f_{z}\right\|>2-\delta .
$$

Therefore, when $\|x-z\|<\delta$

$$
\begin{aligned}
2 & \leqq\left\|f_{x}+f_{z}\right\|+\|x-z\| \\
& <\left\|f_{x}+f_{z}\right\|+\delta,
\end{aligned}
$$

so we conclude that $\left|\left(f_{x}-f_{z}\right)(y)\right|<\varepsilon$. From Theorem 1 we have that the norm of $X$ is uniformly weakly differentiable in the direction $y$.

Conversely, when the norm of $X$ is uniformly weakly differentiable in the direction $y \in S(X)$, it follows that, given $\varepsilon>0$ there exists a $\delta(\varepsilon, y)>0$ such that

$$
\left|\frac{\|x+\lambda y\|-\|x\|}{\lambda}-f_{x}(y)\right|<\varepsilon
$$

and

$$
\left|\frac{\|-x+\lambda y\|-\|x\|}{\lambda}+f_{x}(y)\right|<\varepsilon
$$

for every $x \in S(X)$ when $|\lambda|<\delta$.

Therefore,

$$
\|x+\lambda y\|+\|x-\lambda y\|<2+2 \varepsilon \lambda \quad \text { for every } \mid x \in S(X)
$$

and $0<\lambda<\delta$.

For any $f, g \in S\left(X^{*}\right)$ such that $(f-g)(y) \geqq 3 \varepsilon$ we have

$$
\begin{aligned}
\|f+g\| & =\sup \{(f+g)(x): x \in S(X)\} \\
& =\sup \{f(x+\lambda y)+g(x-\lambda y)-(f-g)(\lambda y): x \in S(X)\} \\
& \leqq \sup \{\|x+\lambda y\|+\|x-\lambda y\|-3 \varepsilon \lambda: x \in S(X)\} \text { for all } \lambda>0, \\
& <2-\varepsilon \delta \text { when } 0<\lambda<\delta .
\end{aligned}
$$


Therefore,

$$
|(f-g)(y)|<3 \varepsilon \text { when }\|f+g\|>2-\varepsilon \delta \text { and so } X^{*} \text { is weak }{ }^{*}
$$

uniformly rotund in the direction $y$.

(ii) It follows from (i) that if the norm of $X^{*}$ is uniformly weakly differentiable in the direction $g \in S\left(X^{*}\right)$ then $X^{* *}$ is weak* uniformly rotund in the direction $g$, and this implies that $X$ is weakly uniformly rotund in the direction $g$.

Conversely, when $X$ is weakly uniformly rotund in the direction $g \in S\left(X^{*}\right)$, then given $\varepsilon>0$ there exists a $\delta(\varepsilon, g)>0$ such that, for $x, y \in S(X)$

$$
|g(x-y)|<\varepsilon \quad \text { when } \quad\|x+y\|>2-\delta .
$$

Therefore, given any support mapping $x \mid \rightarrow f_{x}$ on $X$, when $\left\|f_{x}-f_{y}\right\|<\delta$

$$
2 \leqq\|x+y\|+\left\|f_{x}-f_{y}\right\|<\|x+y\|+\delta,
$$

so we conclude that $|g(x-y)|<\varepsilon$. So the real mapping $f_{x} \mapsto \hat{x}(g)$ is uniformly continuous on $D(X)$. Since $X$ is a Banach space, $D(X)$ is norm dense in $S\left(X^{*}\right)$ so this mapping has a unique continuous extension which is uniformly continuous on $S\left(X^{*}\right)$.

Consider any $f \in S\left(X^{*}\right)$ and a sequence $\left\{f_{x_{n}}\right\}$ where $f_{x_{n}} \in D(X)$ and $\left\{f_{x_{n}}\right\}$ is norm convergent to $f$. Now the sequence $\left\{\hat{x}_{n}\right\}$ has a subnet $\left\{\hat{x}_{n_{\alpha}}\right\}$ weak ${ }^{*}$ convergent to $F \in B\left(X^{* *}\right)$. But

$$
|F(f)-1| \leqq\left|F(f)-\hat{x}_{n_{\alpha}}(f)\right|+\left|f\left(x_{n_{\alpha}}\right)-f_{x_{n_{\alpha}}}\left(x_{n_{\alpha}}\right)\right|,
$$

and so $F \in D(f)$.

Therefore, the extension is a mapping $f \mapsto \rightarrow F_{f}(g)$ which is uniformly continuous on $S\left(X^{*}\right)$. By Theorem 1 we have that the norm of $X^{*}$ is uniformly weakly differentiable in the direction $g$.

Zizler (1968; page 429), investigating isomorphism properties, has shown that every separable Banach space can be equivalently renormed to have norm uniformly weakly differentiable in every direction. This, of course, implies that there are non-reflexive Banach spaces with norm uniformly weakly differentiable in every direction. Such an observation points to the great significance of Vlasov's result for Chebychev sets in that it applies even in non-reflexive spaces.

Zizler (1968; page 427) has also shown that every Banach space with separable dual can be equivalently renormed to have norm weakly uniformly rotund in every direction. From Theorem 2 we see that a Banach space so renormed has second dual weak* uniformly rotund in every direction. However, it is known (Giles (1974)), that a non-reflexive Banach space has non-smooth 
third dual, so a Banach space which has dual weak* uniformly rotund in every direction does not necessarily have smooth second dual.

\section{The Vlasov smoothness condition}

In our discussion of the Vlasov smoothness condition we use geometrical argument associated with two dimensional subspaces of a normed linear space. For a normed linear space $X$, given $a, b \in S(X), a \neq b$, we denote by $X_{a b}$ the Minkowskian plane associated with the normed linear subspace generated by $a$ and $b$. For a choice of $f_{a} \in D(a)$ and $f_{b} \in D(b)$ in $X^{*}$, in $X_{a b}$ we denote by $T_{a}$ and $T_{b}$ the tangent lines, $\left\{x \in X_{a b}: f_{a}(x)=1\right\}$ and $\left\{x \in X_{a b}: f_{b}(x)=1\right\}$, to $S\left(X_{a b}\right)$ at $a$ and $b$. If $T_{a}$ or $T_{b}$ meet the straight line through 0 parallel to $a-b$, we denote the points of intersection by $p$ and $q$.

We make use of the following general geometrical property associated with support mappings on a normed linear space. A similar result has been given by Browder (1965; page 370).

Lemma 1. Given a support mapping $x \mapsto f_{x}$ on $X$ and $a, b \in S(X), a \neq b$, if in $X_{a b}$ the ray through $c_{\lambda} \equiv \lambda a+(1-\lambda) b$ for $0<\lambda<1$ meets $S\left(X_{a b}\right)$ in $d_{\lambda}$, then

$$
\left(f_{a}-f_{b}\right)(a-b) \geqq \Delta \equiv 2 \sup \left\{\left\|c_{\lambda}-d_{\lambda}\right\|: 0<\lambda<1\right\} .
$$

Proof. Case 1: Suppose that $c_{\lambda} \notin S\left(X_{a b}\right)$ for some $0<\lambda<1$. Then $p$ and $q$ are defined. Let $T_{a}$ and $T_{b}$ meet the ray through $c_{\lambda}$ in $a_{\lambda}$ and $b_{\lambda}$.

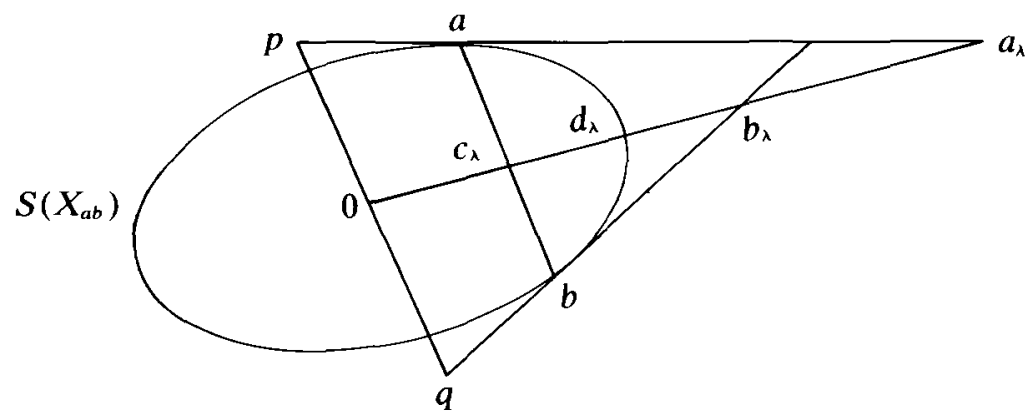

Figure 1.

Now

$$
f_{a}(a-b)=\frac{\|a-b\|}{\|p\|}
$$

and

$$
f_{b}(b-a)=\frac{\|a-b\|}{\|q\|} .
$$


We have

$$
\begin{aligned}
& \frac{\left\|a-c_{\lambda}\right\|}{\|p\|}=\frac{\left\|a_{\lambda}-c_{\lambda}\right\|}{1-\left\|c_{\lambda}-d_{\lambda}\right\|+\left\|a_{\lambda}-c_{\lambda}\right\|} \\
&>\left\|c_{\lambda}-d_{\lambda}\right\|, \quad \text { and similarly } \\
& \frac{\left\|b-c_{\lambda}\right\|}{\|q\|}>\left\|c_{\lambda}-d_{\lambda}\right\| .
\end{aligned}
$$

Therefore,

$$
\left(f_{a}-f_{b}\right)(a-b)>2\left\|c_{\lambda}-d_{\lambda}\right\|
$$

Case 2: Suppose that $c_{\lambda} \in S\left(X_{a b}\right)$ for all $0<\lambda<1$. Then $\Delta=0$. If $f_{a}$ has been chosen so that $b \in T_{a}$ then $f_{a}(a-b)=0$. A similar remark could be made for $f_{b}$. But again the result holds.

Corollary for CASE 1.

$$
\|p-q\|<\frac{2\|a-b\|}{\Delta}
$$

Proof. We have from the proof of Lemma 1, that

and

$$
\begin{aligned}
& \|p\|<\frac{\|a-b\|}{\left\|c_{\lambda}-d_{\lambda}\right\|}, \\
& \|q\|<\frac{\|a-b\|}{\left\|c_{\lambda}-d_{\lambda}\right\|} \text { for all } 0<\lambda<1 .
\end{aligned}
$$

We now define the Vlasov smoothness condition and derive equivalent reformulations of it which give some geometrical insight into the nature of the condition and which reveal it as a differentiability of the norm condition related to uniformly weak differentiability of the norm.

THEOREM 3. In a normed linear space $X$ for a given $y \in S(X)$ the following conditions are equivalent.

(i) The uniform chord condition in the direction $y$.

Given $\varepsilon>0$ there exists a $\delta(\varepsilon, y)>0$ and a support mapping $x \mapsto f_{x}$ on $X$ such that for all $a, b \in S(X), a \neq b$, where $a-b$ is parallel to $y$,

$$
\min \left\{f_{a}\left(\frac{a-b}{\|a-b\|}\right), \quad f_{b}\left(\frac{b-a}{\|a-b\|}\right)\right\}<\varepsilon
$$

when $\|a-b\|<\delta$.

(ii) The Vlasov smoothness condition in the direction $y$.

For given $d, e \in X, d \neq e$, where $d-e$ is parallel to $y$, and given $\varepsilon>0$ there exists an $R>0$ such that for any $z \in X$ where $\|z-(d+e) / 2\|=R$ we have

$$
\min \{\|z-d\|,\|z-e\|\}<\varepsilon+R \text {. }
$$


(iii) One sided uniformly weak differentiability of the norm in the direction $y$.

Given $\varepsilon>0$ there exists a $\delta>0$ such that, for all

$$
\begin{gathered}
x \in y^{\perp} \equiv\{x \in S(X):\|x+\lambda y\|-\|x\| \geqq 0 \text { for all } \lambda\}, \\
\min \left\{\frac{\|x+\lambda y\|-\|x\|}{\lambda}, \frac{\|x-\lambda y\|-\|x\|\}}{\lambda}\right\}<\varepsilon
\end{gathered}
$$

when $0<\lambda<\delta$.

Before proceeding with the proof of this theorem we should explain that the name "uniform chord condition" signifies the geometrical interpretation of property (i). When $p$ and $q$ are defined then

$$
f_{a}\left(\frac{a-b}{\|a-b\|}\right)=\frac{1}{\|p\|} \text { and } f_{b}\left(\frac{b-a}{\|a-b\|}\right)=\frac{1}{\|q\|},
$$

and so property (i) is equivalent to

$$
\min \left\{\frac{1}{\|p\|}, \frac{1}{\|q\|}\right\}<\varepsilon \quad \text { when }\|a-b\|<\delta .
$$

So property (i) has the following geometrical interpretation.

For all given $a, b \in S(X), a \neq b$, where $a-b$ is parallel to $y$, and when $p$ and $q$ are defined, for given $R>0$ there exists a $\delta>0$ such that

$$
\|p-q\|>R \text { when }\|a-b\|<\delta .
$$

Proof. (i) $\Rightarrow$ (ii).

Suppose that $X$ does not have the Vlasov smoothness condition in the direction $y$. Then for a given $d, e \in X, d \neq e$, where $d-e$ is parallel to $y$, and for some $\varepsilon_{1}>0$ and any $R>0$ there exists a $z \in X$ where $\|z-(d+e) / 2\|=R$ and

and

$$
\varepsilon_{1}+\left\|z-\frac{d+e}{2}\right\| \leqq\|z-d\|
$$

$$
\varepsilon_{1}+\left\|z-\frac{d+e}{2}\right\| \leqq\|z-e\| .
$$

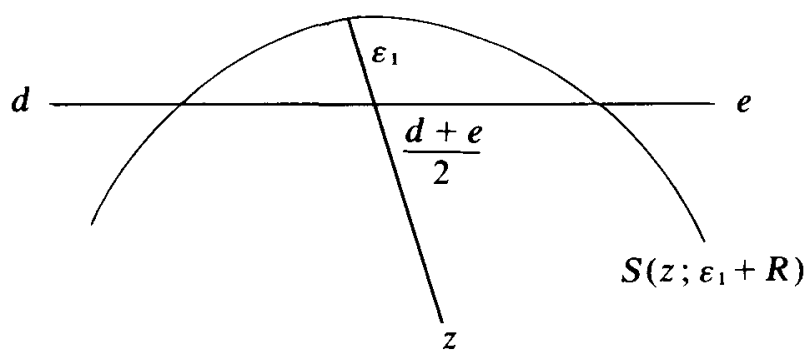

Figure 2. 
Consider the similarity transformation taking $B\left[z ; \varepsilon_{1}+R\right]$ to $B(X)$. Then

$$
d^{\prime} \equiv \frac{d-z}{\varepsilon_{1}+R} \notin B(X), \quad e^{\prime} \equiv \frac{e-z}{\varepsilon_{1}+R} \notin B(X)
$$

but

$$
\left(\frac{d+e}{2}\right)^{\prime} \equiv \frac{\frac{d+e}{2}-z}{\varepsilon_{1}+R} \in B(X) .
$$

There exist $d^{\prime \prime}, e^{\prime \prime} \in S\left(X_{d^{\prime} e^{\prime}}\right), d^{\prime \prime} \neq e^{\prime \prime}$, where the straight line defined by $d^{\prime}$ and $e^{\prime}$ in $X_{d^{\prime} e^{\prime}}$ meets $S\left(X_{d^{\prime} e^{\prime}}\right)$. Denoting by $p^{\prime \prime}$ and $q^{\prime \prime}$ the points of intersection of the tangent lines $T_{\mathrm{d}^{\prime \prime}}$ and $T_{e^{\prime \prime}}$ with the straight line through 0 parallel to $y$, we deduce from the Corollary for Case 1 of Lemma 1 that $\left\|p^{\prime \prime}-q^{\prime \prime}\right\|<2(\|d-e\|) / \varepsilon_{1}$, although $\left\|d^{\prime \prime}-e^{\prime \prime}\right\| \rightarrow 0$ as $R \rightarrow \infty$. So we conclude that $X$ does not have the uniform chord condition in the direction $y$.

(ii) $\Rightarrow$ (iii).

For given $d, e \in X, d \neq e$, where $d-e$ is parallel to $y$, and given $\varepsilon>0$ and $z \in X$ satisfying the Vlasov smoothness condition, consider the similarity transformation taking $B[z ; R]$ to $B(X)$. Then writing

$$
x \equiv \frac{\frac{d+e}{2}-z}{R}, \quad y \equiv \frac{d-e}{2 \| \frac{d-e \|}{d}} \quad \text { and } \quad \lambda \equiv \frac{\|d-e\|}{R},
$$

we have that the Vlasov smoothness condition in the direction $y$ takes the following form.

Given $\varepsilon>0$ there exists a $\lambda>0$ such that for all $x \in S(X)$ we have

$$
\min \left\{\frac{\|x+\lambda y\|-\|x\|}{\lambda}, \frac{\|x-\lambda y\|-\|x\|}{\lambda}\right\} \leqq \frac{\varepsilon}{\|d-e\|} .
$$

Using the monotonicity of $(\|x+\lambda y\|-\|x\|) / \lambda$ as a function of $\lambda$ we deduce that $X$ has one sided uniformly weak differentiability of the norm in the direction $y$.

(iii) $\Rightarrow$ (i)

For a given $x \in y^{\perp}$, consider $a, b \in S\left(X_{x y}\right), a \neq b$ where $a-b$ is parallel to $y$. Suppose that the ray through 0 and $a$ meets the straight line through $x$ parallel to $y$ in $x+\lambda y$ and the straight line through $a$ and $b$ meets the ray through 0 and $x$ in $c$.

Working in $X_{x y}$ we deduce that

$$
\lambda=\|x+\lambda y\|\|a-c\| .
$$




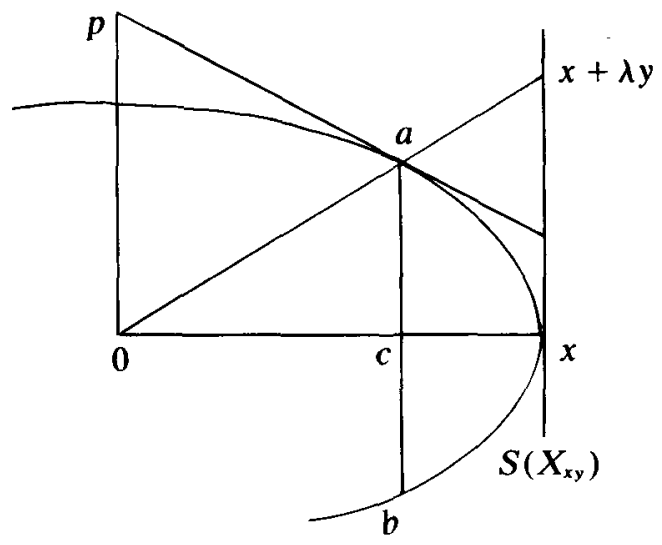

Figure 3.

Then

$$
\frac{\|x+\lambda y\|-\|x\|}{\lambda}=\frac{1}{\|a-c\|}-\frac{1}{\lambda} .
$$

But also we have

$$
\frac{1}{\|p\|}>\frac{\|x+\lambda y\|-\|x\|}{\lambda},
$$

and therefore,

$$
\lambda<\frac{\|p\|\|a-c\|}{\|p\|-\|a-c\|}
$$

Suppose that $X$ does not have the uniform chord condition in the direction $y$. Then there exists an $r>0$ and a sequence $\left\{x_{n}\right\}$ where $x_{n} \in y^{\perp}$, and sequences $\left\{a_{n}\right\},\left\{b_{n}\right\}$ where $a_{n}, b_{n} \in S\left(X_{x y}\right)$ and $a_{n}-b_{n}$ is parallel to $y$ and $\left\|a_{n}-b_{n}\right\|<1 / n$ but also $\left\|p_{n}-q_{n}\right\|<r$, where $p_{n}, q_{n}$ denote the points of intersection of the tangent lines $T_{a_{n}}, T_{b_{n}}$ with the straight line through 0 parallel to $y$. Suppose that the rays through 0 and $a_{n}$, and 0 and $b_{n}$ meet the straight line through $x_{n}$ parallel to $y$ in points $x_{n}+\lambda_{n} y$ and $x_{n}-\lambda_{n}^{\prime} y$. We note from the inequalities $(*)$ above, that for given $0<\varepsilon<1 / r$,

only if

$$
\frac{\left\|x_{n}+\lambda_{n} y\right\|-\left\|x_{n}\right\|}{\lambda_{n}}<\varepsilon<\frac{1}{r}<\frac{1}{\left\|p_{n}\right\|}
$$

$$
\lambda_{n}<\frac{\left\|p_{n}\right\|\left\|a_{n}-c_{n}\right\|}{\left\|p_{n}\right\|-\left\|a_{n}-c_{n}\right\|}<\frac{r}{n-1}
$$

Now denote by $b_{n}^{\prime}$ the point of intersection of the ray through 0 and $x_{n}-\lambda_{n} y$ with $S\left(X_{x_{n} y}\right)$. Denote by $c_{n}^{\prime}$ the point of intersection of the ray through 0 and $x_{n}$ with the straight line through $b_{n}^{\prime}$ parallel to $y$. 


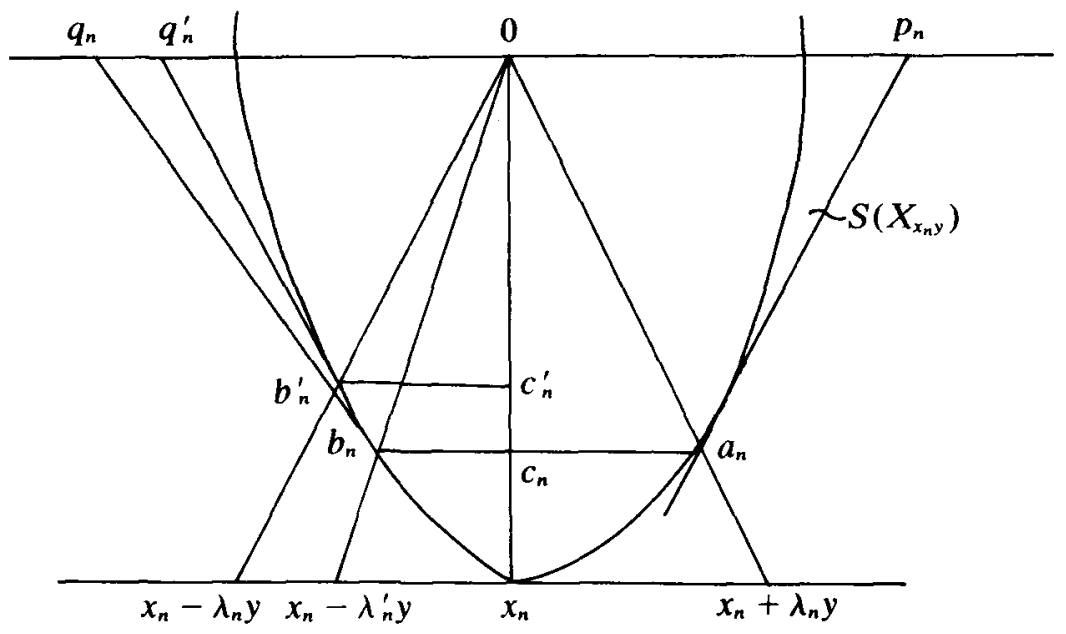

Figure 4 .

We may assume that $\lambda_{n}>\lambda_{n}^{\prime}$, for which it follows that $\left\|q_{n}^{\prime}\right\| \leqq\left\|q_{n}\right\|<r$ where $q_{n}^{\prime}$ denotes the point of intersection of the tangent line $T_{b_{n}^{\prime}}$ with the straight line through 0 parallel to $y$.

Working in $X_{x_{n} y}$ we have

$$
\lambda_{n}=\left\|a_{n}-c_{n}\right\|\left\|x_{n}+\lambda_{n} y\right\|<\frac{2}{n} \text { for } \lambda_{n}<1 .
$$

But also

$$
\lambda_{n}=\left\|b_{n}^{\prime}-c_{n}^{\prime}\right\|\left\|x_{n}-\lambda_{n} y\right\| \geqq\left\|b_{n}^{\prime}-c_{n}^{\prime}\right\|,
$$

so

$$
\left\|b_{n}^{\prime}-c_{n}^{\prime}\right\|<\frac{2}{n} .
$$

We have again from the inequalities $(*)$ above, that

$$
\frac{\left\|x_{n}-\lambda_{n} y\right\|-\left\|x_{n}\right\|}{\lambda_{n}}<\varepsilon<\frac{1}{r}<\frac{1}{\left\|q_{n}^{\prime}\right\|}
$$

only if

$$
\lambda_{n}<\frac{\left\|q_{n}^{\prime}\right\|\left\|b_{n}^{\prime}-c_{n}^{\prime}\right\|}{\left\|q_{n}^{\prime}\right\|-\left\|b_{n}^{\prime}-c_{n}^{\prime}\right\|}<\frac{2 r}{n-2}
$$

But (i) and (ii) give us that there does not exist a $\delta>0$ for all $x \in y^{\perp}$ to satisfy sne sided uniformly weak differentiability of the norm in the direction $y$.

The result given by Vlasov (1967; page 402) can easily be deduced from orms (iii) or (i) of Theorem 3. 
COROLlary. If the norm of $X$ is uniformly weakly differentiable in the direction $y \in S(X)$ then $X$ satisfies the Vlasov smoothness condition in the direction $y$.

However, the converse of this corollary is not true in general; the Vlasov smoothness condition in a direction is a weaker property than uniformly weak differentiability of the norm in that direction.

EXAMPLE 1. Consider $\mathbf{R}^{2}$ with norm generated from the unit sphere given by the graph of the mapping

$$
f(\alpha)=\left\{\begin{array}{lr}
\frac{1}{2} & -1 \leqq \alpha \leqq 0 \\
-\frac{1}{2}+\sqrt{1-\alpha^{2}} & 0<\alpha \leqq 1
\end{array}\right.
$$

completed by symmetry about 0 .

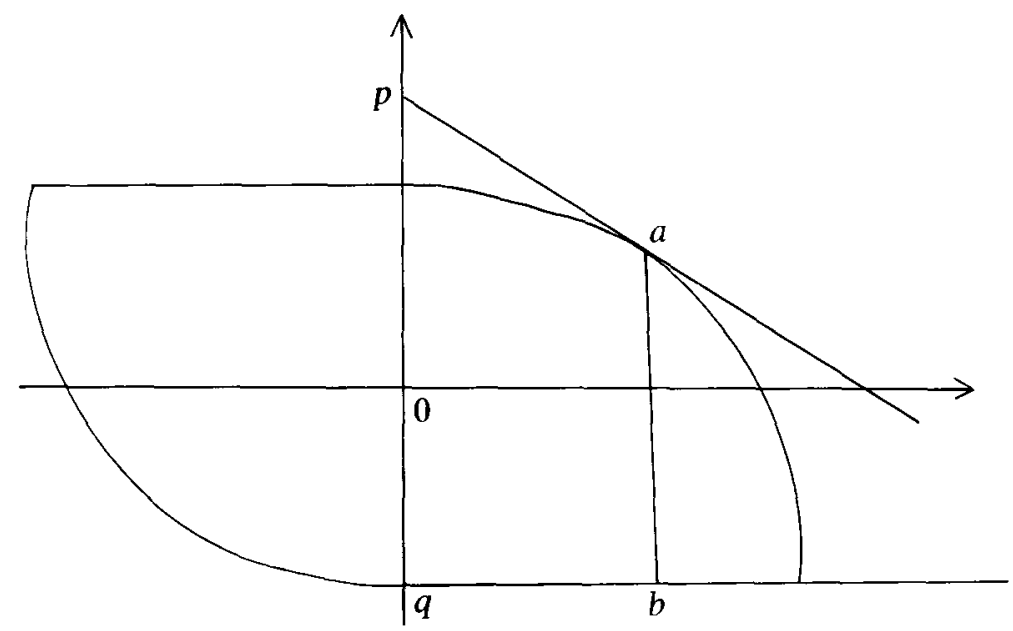

Figure 5.

Consider $a, b \in S(X), a \neq b$ where $a-b$ is parallel to the $Y$-axis. Now $\|p-q\| \rightarrow \infty$ as $\|a-b\| \rightarrow 0$. But $\|q\|=1$, so

$$
\left(f_{a}-f_{b}\right)\left(\frac{a-b}{\|a-b\|}\right)=\frac{1}{\|p\|}+\frac{1}{\|q\|}>1
$$

for all such $a, b \in S(X)$, although we may have $\|a-b\| \rightarrow 0$.

Yet it is clear that if $X$ has the Vlasov smoothness condition in every direction then $X$ is smooth. 
In his later paper Vlasov (1970; page 776) gave the following important characterisation of rotundity of the dual of a normed linear space.

Lemma 2. For a normed linear space $X$, the dual space $X^{*}$ is rotund if and only if for any sequence of closed balls $\left\{B_{n}\right\}, B_{n} \equiv B\left[z_{n}, R_{n}\right]$, where $B_{n} \subseteq B_{n+1}$ for all $n$, and $R_{n} \rightarrow \infty$, then $\cup B_{n}$ is either $X$ or a half-space of $X$.

We use this lemma to derive the following duality relation, which establishes that Vlasov's result (1967; page 403) is included in his result (1970; page 778).

THEOREM 4. If a normed linear space $X$ satisfies the Vlasov smoothness condition in every direction then its dual $X^{*}$ is rotund.

Proof. If $X^{*}$ is not rotund then from Lemma 2, we have that there exists a sequence of closed balls $\left\{B_{n}\right\}, B_{n} \equiv B\left[z_{n}, R_{n}\right]$, where $B_{n} \subseteq B_{n+1}$ for all $n$, and $R_{n} \rightarrow \infty$, but where $\overline{\cup B_{n}}$ is not $X$ and is not a half-space. It is clear from the separation properties of convex sets that there exist linearly independent continuous linear functionals $f$ and $g$ such that

$$
\overline{\cup B_{n}} \subseteq f^{-1}[0, \infty) \cap g^{-1}[0, \infty) .
$$

However, since $\overline{\cup B_{n}} \subseteq \operatorname{co}\left\{f^{-1}(0), g^{-1}(0)\right\}$ we see that there exists $d, e \notin \overline{\cup B_{n}}$ but $(d+e) / 2=z_{1}$. For any given $n$, consider the similarity transformation taking $B_{n}$ to $B(X)$. Then

$$
d^{\prime} \equiv \frac{d-z_{n}}{R_{n}} \notin B(X), \quad e^{\prime} \equiv \frac{e-z_{n}}{R_{n}} \notin B(X)
$$

but

$$
\left(\frac{d+e}{2}\right)^{\prime} \equiv \frac{\frac{d+e}{2}-z_{n}}{R_{n}} \in B(X)
$$

There exist $d^{\prime \prime}, e^{\prime \prime} \in S\left(X_{d^{\prime} e^{\prime}}\right), d^{\prime \prime} \neq e^{\prime \prime}$, where the straight line defined by $d^{\prime}$ and $e^{\prime}$ in $X_{d^{\prime} e^{\prime}}$ meets $S\left(X_{d^{\prime} e^{\prime}}\right)$. Denoting by $p^{\prime \prime}$ and $q^{\prime \prime}$ the points of intersection of the tangent lines $T_{d^{\prime \prime}}$ and $T_{e^{\prime \prime}}$ with the straight line through 0 parallel to $d-e$, we deduce from the Corollary for Case 1 of Lemma 1 that $\left\|p^{\prime \prime}-q^{\prime \prime}\right\|<(\|d-e\|) / R_{1}$ although $\left\|d^{\prime \prime}-e^{\prime \prime}\right\| \rightarrow 0$ as $R_{n} \rightarrow \infty$. So we conclude that $X$ does not have the uniform chord property in the direction $d-e$.

Now there exists a smooth normed linear space $X$ where the dual $X^{*}$ is not rotund (Cudia (1964; page 289)), so this theorem shows that not every smooth normed linear space satisfies the Vlasov smoothness condition in every direction.

For a normed linear space $X$ we say that the dual space $X^{*}$ has the Vlasov 
rotundity condition in the direction $y \in S(X)$ if given $\varepsilon>0$ there exists a $\delta(\varepsilon, y)>0$ and a support mapping $x \mapsto \rightarrow f x$ on $X$ such that for all $a, b \in S(X)$, $a \neq b$, where $a-b$ is parallel to $y$,

$$
\min \left\{f_{a}\left(\frac{a-b}{\|a-b\|}\right), \quad f_{b}\left(\frac{b-a}{\|a-b\|}\right)\right\}<\varepsilon
$$

when $\left\|f_{a}+f_{b}\right\|>2-\delta$.

We now establish a duality relation for the Vlasov smoothness and rotundity conditions similar to that given in Theorem 2(i) for uniformly weak differentiability of the norm and weak* uniform rotundity.

Theorem 5. A normed linear space $X$ satisfies the Vlasov smoothness condition in the direction $y \in S(X)$ if and only if its dual $X^{*}$ satisfies the Vlasov rotundity condition in the direction $y$.

Proof. Using the primitive parallelogram law as in Theorem 2(i) it is evident that when $X^{*}$ has the Vlasov rotundity condition in the direction $y$ then $X$ has the uniform chord condition in the direction $y$.

Conversely, when $X$ has the Vlasov smoothness condition in the direction $y$ we have from the proof (ii) $\Rightarrow$ (iii) in Theorem 3, that given $\varepsilon>0$ there exists a $\delta(\varepsilon, y)>0$ such that for all $x \in S(X)$

$$
\min \{\|x+\lambda y\|,\|x-\lambda y\|\}<1+\varepsilon \delta
$$

when $0<\lambda<\delta$.

For any $f_{a}, f_{b}$ such that both

we have

$$
f_{a}\left(\frac{a-b}{\|a-b\|}\right) \text { and } f_{b}\left(\frac{b-a}{\|a-b\|}\right) \geqq 3 \varepsilon,
$$

$$
\begin{aligned}
& \left\|f_{a}+f_{b}\right\|=\sup \left\{\left(f_{a}+f_{b}\right)(x): x \in S(X)\right\} \\
& \leqq \min \left\{\begin{array}{l}
\sup \left\{f_{a}(x+\lambda y)+f_{b}(x)-f_{a}(\lambda y): x \in S(X)\right\} \\
\sup \left\{f_{a}(x)+f_{b}(x-\lambda y)+f_{b}(\lambda y): x \in S(X)\right\}
\end{array}\right. \\
& \leqq \min \left\{\begin{array}{l}
\sup \left\{\|x+\lambda y\|+\|x\|-\lambda f_{a}\left(\frac{a-b}{\|a-b\|}\right): x \in S(X)\right\} \\
\sup \left\{\|x\|+\|x-\lambda y\|-\lambda f_{b}\left(\frac{b-a}{\| a-b}\right): x \in S(X)\right\}
\end{array}\right. \\
& <2-\varepsilon \lambda .
\end{aligned}
$$


Therefore,

$$
\min \left\{f_{a}\left(\frac{a-b}{\|a-b\|}\right), f_{b}\left(\frac{b-a}{\|a-b\|}\right)\right\}<3 \varepsilon \quad \text { when }\left\|f_{a}+f_{b}\right\|>2-\varepsilon \delta .
$$

We use this Vlasov rotundity for the dual space to provide a counterexample to the converse of Theorem 4; that is, to show that a Banach space with rotund dual does not necessarily have the Vlasov smoothness condition in every direction.

Example 2. Consider Hilbert space $l_{2}$ with natural basis $\left\{e_{n}\right\}$. Brown (1974; page 146) has shown that it is possible to give $l_{2}$ an equivalent rotund norm such that for each $k \geqq 2$ the two dimensional subspaces $M_{k} \equiv \operatorname{sp}\left\{e_{1}, e_{k}\right\}$ have an $l_{k}$ norm; that is, for $x \equiv\left\{\alpha_{1}, 0, \cdots, \alpha_{k}, 0, \cdots\right\}$

$$
\|x\|=\left(\left|\alpha_{1}\right|^{k}+\left|\alpha_{k}\right|^{k}\right)^{1 / k} \text {. }
$$

Since $l_{2}$ with this norm is reflexive we will treat it as a dual space and show that it does not have the Vlasov rotundity condition in the direction $e_{1}$. For each $k \geqq 2$ consider $f_{a_{k}}, f_{b_{k}} \in M_{k}$ where

and

$$
f_{a_{k}}=\left(\left(\frac{1}{k}\right)^{1 / k},\left(1-\frac{1}{k}\right)^{1 / k}\right)
$$

$$
f_{b_{k}}=\left(-\left(\frac{1}{k}\right)^{1 / k},\left(1-\frac{1}{k}\right)^{1 / k}\right)
$$

Now working in the subspaces $M_{k}$ we have for $f \equiv\left(\alpha_{1}, \alpha_{k}\right)$,

and

$$
\hat{a}_{k}(f)=\left(\frac{1}{k}\right)^{1-1 / k} \alpha_{1}+\left(1-\frac{1}{k}\right)^{1-1 / k} \alpha_{k}
$$

$$
\hat{b}_{k}(f)=-\left(\frac{1}{k}\right)^{1-1 / k} \alpha_{1}+\left(1-\frac{1}{k}\right)^{1-1 / k} \alpha_{k}
$$

So

$$
\left(\hat{a}_{k}-\hat{b}_{k}\right)(f)=2\left(\frac{1}{k}\right)^{1-1 / k} \alpha_{1}
$$

Working with the whole space we have

$$
\left\|\hat{a}_{k}-\hat{b}_{k}\right\|=2\left(\frac{1}{k}\right)^{1-1 / k}
$$

and

$$
f_{a_{k}}\left(\frac{a_{k}-b_{k}}{\left\|a_{k}-b_{k}\right\|}\right)=\left(\frac{1}{k}\right)^{1 / k} \rightarrow 1
$$


and similarly

$$
f_{b_{k}}\left(\frac{b_{k}-a_{k}}{\left\|a_{k}-b_{k}\right\|}\right)=\left(\frac{1}{k}\right)^{1 / k} \rightarrow 1 \text { as } k \rightarrow \infty
$$

However,

$$
\left\|f_{a_{k}}+f_{b_{k}}\right\|=2\left(1-\frac{1}{k}\right)^{1 / k} \rightarrow 2 \text { as } k \rightarrow \infty .
$$

This example also shows that there exist reflexive smooth Banach spaces which do not satisfy the Vlasov smoothness condition in every direction. It also verifies that Vlasov's 1970 result does cover more cases than his 1967 result.

It is possible to modify Example 2 to strengthen the evidence of Example 1 and show that even for a reflexive smooth Banach space, the Vlasov smoothness condition in a direction is a weaker property than uniformly weak differentiability of the norm in that direction.

EXAmple 3. Consider again Hilbert space $l_{2}$ with natural basis $\left\{e_{n}\right\}$. Using the method outlined by Brown (1974) it is possible to give $l_{2}$ an equivalent rotund norm such that for each $k \geqq 2$ the dimensional subspaces $M_{k} \equiv \operatorname{sp}\left\{e_{1}, e_{k}\right\}$ have, in the first and third quadrants an $l_{k}$ norm and in the second and fourth quadrants the $l_{2}$ norm; that is, for $x \equiv\left\{\alpha_{i}, 0, \cdots, \alpha_{k}, 0, \cdots\right\}$

$$
\begin{aligned}
\|x\| & =\left(\left|\alpha_{1}\right|^{k}+\left|\alpha_{k}\right|^{k}\right)^{1 / k} \text { for } \alpha_{i} \alpha_{k} \geqq 0 \\
& =\left(\left|\alpha_{1}\right|^{2}+\left|\alpha_{k}\right|^{2}\right)^{1 / 2} \text { for } \alpha_{1} \alpha_{k} \leqq 0 .
\end{aligned}
$$

Again since $l_{2}$ with this norm is reflexive we will treat it as a dual space and show that it is not weakly uniformly rotund in the direction $e_{1}$ but appears to satisfy the Vlasov rotundity condition in the direction $e_{1}$. For each $k \geqq 2$ consider $f_{a_{k}}, f_{b_{k}} \in M_{k}$ where

$$
\begin{gathered}
f_{a_{k}} \equiv\left(\left(\frac{1}{k}\right)^{1 / k},\left(1-\frac{1}{k}\right)^{1 / k}\right) \\
f_{b_{k}} \equiv\left(-\left(1-\left(1-\frac{1}{k}\right)^{2-2 / k}\right)^{1 / 2},\left(1-\frac{1}{k}\right)^{1-1 / k}\right) .
\end{gathered}
$$

Now working in the subspace $M_{k}$ we have for $f \equiv\left(\alpha_{1}, \alpha_{k}\right)$

and

$$
\hat{a}_{k}(f)=\left(\frac{1}{k}\right)^{1-1 / k} \alpha_{1}+\left(1-\frac{1}{k}\right)^{1-1 / k} \alpha_{k}
$$

$$
\hat{b}_{k}(f)=-\left(1-\left(1-\frac{1}{k}\right)^{2-2 / k}\right)^{1 / 2} \alpha_{1}+\left(1-\frac{1}{k}\right)^{1-1 / k} \alpha_{k}
$$


So

$$
\left(\hat{a}_{k}-\hat{b}_{k}\right)(f)=\left(\left(\frac{1}{k}\right)^{1-1 / k}+\left(1-\left(1-\frac{1}{k}\right)^{2-2 / k}\right)^{1 / 2}\right) \alpha_{1} .
$$

Working with the whole space we have

$$
f_{a_{k}}\left(\frac{a_{k}-b_{k}}{\left\|a_{k}-b_{k}\right\|}\right)=\left(\frac{1}{k}\right)^{1 / k} \rightarrow 1 \quad \text { as } \quad k \rightarrow \infty
$$

and

Now

$$
f_{b_{k}}\left(\frac{b_{k}-a_{k}}{\left\|a_{k}-b_{k}\right\|}\right)=\left(1-\left(1-\frac{1}{k}\right)^{2-2 / k}\right)^{1 / 2} \rightarrow 0 \text { as } k \rightarrow \infty .
$$

Therefore,

$$
\left\|f_{a_{k}}+f_{b_{k}}\right\| \rightarrow 2 \text { as } k \rightarrow \infty .
$$

but

$$
\min \left\{f_{a_{k}}\left(\frac{a_{k}-b_{k}}{\left\|a_{k}-b_{k}\right\|}\right), f_{b_{k}}\left(\frac{b_{k}-a_{k}}{\left\|a_{k}-b_{k}\right\|}\right)\right\} \rightarrow 0
$$

$$
\left(f_{a_{k}}-f_{b_{k}}\right)\left(\frac{a_{k}-b_{k}}{\left\|a_{k}-b_{k}\right\|}\right) \nrightarrow 0 \text { as }\left\|f_{a_{k}}+f_{b_{k}}\right\| \rightarrow 2 .
$$

\section{References}

F. E. Browder (1965), 'On a theorem of Beurling and Livingston', Canad. J. Math. 17, 367-372.

A. L. Brown (1974), 'A rotund reflexive space having a subspace of codimension two with a discontinuous metric projection', Michigan Math. J. 21, 145-151.

D. F. Cudia (1964), 'The geometry of Banach spaces. Smoothness', Trans. Amer. Math. Soc. 110, 284-314.

J. R. Giles (1971), 'On a characterisation of differentiability of the norm of a normed linear space', $J$. Austral. Math. Soc. 12, 106-114.

J. R. Giles (1974), 'A non-reflexive Banach space has non-smooth third conjugate space', Canad. Math. Bull. 17, 117-119.

V. L. Klee (1961), 'Convexity of Chebychev sets', Math. Ann. 142, 292-304.

V. L. Šmulian (1940), 'Sur la dérivabilité de la norme dans l'espace de Banach', Dokl. Akad. Nauk. SSSR (N. S.) 27, 643-648.

L. P. Vlasov (1967), 'On Chebychev sets', Soviet Math. Dokl. 8, 401-404.

L. P. Vlasov (1970), 'Almost convex and Chebychev sets', Math. Notes. Acad. Sc. USSR. 8, 776-779.

V. Zizler (1968), 'Banach spaces with differentiable norms', Comm. Math. Univ. Carol. 8, 3, 415-440.

University of Newcastle,

New South Wales, 2308,

Australia. 